\title{
Permutations without long decreasing subsequences and random matrices
}

\author{
Piotr Śniady \\ Institute of Mathematics \\ University of Wroclaw \\ pl. Grunwaldzki 2/4 \\ 50-384 Wroclaw, Poland \\ Piotr.Sniady@math.uni.wroc.pl
}

Submitted: Jan 8, 2006; Accepted: Dec 31, 2006; Published: Jan 10, 2007

Mathematics Subject Classifications: 05E10, 15A52, 60J65

\begin{abstract}
We study the shape of the Young diagram $\lambda$ associated via the RobinsonSchensted-Knuth algorithm to a random permutation in $S_{n}$ such that the length of the longest decreasing subsequence is not bigger than a fixed number $d$; in other words we study the restriction of the Plancherel measure to Young diagrams with at most $d$ rows. We prove that in the limit $n \rightarrow \infty$ the rows of $\lambda$ behave like the eigenvalues of a certain random matrix (namely the traceless Gaussian Unitary Ensemble random matrix) with $d$ rows and columns. In particular, the length of the longest increasing subsequence of such a random permutation behaves asymptotically like the largest eigenvalue of the corresponding random matrix.
\end{abstract}

\section{Introduction}

\subsection{Formulation of the problem}

Let an integer $d \geq 1$ be fixed. For any integer $n \geq 1$ we consider the set of the permutations $\pi \in S_{n}$ such that the length of the longest decreasing subsequence of $\pi$ is not bigger than $d$; in other words it is the set of the permutations avoiding the pattern $(d+1, d, \ldots, 3,2,1)$. Let $\pi_{n}$ be a random element of this set (probabilities of all elements are equal). In this article we are interested in the following problem:

Problem 1. Let $\pi_{n} \in S_{n}$ be a random permutation with the longest decreasing subsequence of length at most $d$. What can we say about the asymptotic behavior of the length of the longest increasing subsequence of $\pi_{n}$ in the limit $n \rightarrow \infty$ ? 
Let $\lambda_{n}=\left(\lambda_{n, 1}, \ldots, \lambda_{n, d}\right)$ be the (random) Young diagram associated via the RobinsonSchensted-Knuth algorithm to $\pi_{n}$ (notice that since the number of the rows of $\lambda_{n}$ is equal to the length of the longest decreasing subsequence of $\pi_{n}, \lambda_{n}$ has at most $d$ rows). In other words, $\lambda_{n}$ is a random Young diagram with at most $d$ rows, where the probability of the Young diagram $\lambda$ is proportional to $\left(\operatorname{dim} \rho_{\lambda}\right)^{2}$, where $\operatorname{dim} \rho_{\lambda}$ denotes the dimension of the corresponding irreducible representation of $S_{n}$; therefore, if we drop the restriction on the number of the rows of the Young diagrams (which can be alternatively stated as $d \geq n$ ), then the distribution of $\lambda_{n}$ is the celebrated Plancherel measure.

Since $\lambda_{n, 1}$ is equal to the length of the longest increasing subsequence in $\pi_{n}$, Problem 1 is a special case of the following more general one:

Problem 2. What can we say about the asymptotic behavior of the random variables $\left(\lambda_{n, 1}, \ldots, \lambda_{n, d}\right)$ in the limit $n \rightarrow \infty$ ?

\subsection{Case $d=2$}

The first non-trivial case $d=2$ was considered by Deutsch, Hildebrand and Wilf [DHW03]. In this case the random variables $\lambda_{n, 1}, \lambda_{n, 2}$ are subject to a constraint $\lambda_{n, 1}+\lambda_{n, 2}=n$ therefore it is enough to study the distribution of $\lambda_{n, 1}$. Deutsch, Hildebrand and Wilf proved that the distribution of $\sqrt{\frac{8}{n}}\left(\lambda_{n, 1}-\frac{n}{2}\right)$ converges to the distribution of the length of a random Gaussian vector in $\mathbb{R}^{3}$; in other words $\frac{8}{n}\left(\lambda_{n, 1}-\frac{n}{2}\right)^{2}$ converges to the $\chi_{3}^{2}$ distribution with 3 degrees of freedom (a careful reader may notice that the authors of DHW03 use a non-standard definition of the $\chi^{2}$ distributions and therefore they claim that $\sqrt{\frac{8}{n}}\left(\lambda_{n, 1}-\frac{n}{2}\right)$ itself converges to $\left.\chi_{3}^{2}\right)$. Their proof was based on an explicit calculation of the number of the permutations which correspond to a prescribed Young diagram with at most two rows.

\subsection{Case $d=\infty$}

Another extreme of this problem is to consider $d=\infty$; in other words, not to impose any restrictions on the random permutations $\pi_{n}$. In this case the random Young diagram $\lambda_{n}$ is distributed according to the Plancherel measure. The authors of [BDJ99, Oko00, Joh01] proved that the joint distribution of longest rows of $\lambda$ (after appropriate rescaling) converges to the same distribution (called Tracy-Widom distribution) as the joint distribution of the biggest eigenvalues of a large random matrix from the Gaussian Unitary Ensemble.

\subsection{The main result: intermediate values of $d$}

We equip the vector space of $d \times d$ Hermitian matrices with a Gaussian probability measure with a density

$$
\frac{1}{Z_{d}} e^{-\frac{1}{2} \operatorname{Tr} H^{2}}
$$


with respect to the Lebesgue measure, where $Z_{d}$ is the normalizing constant. We say that a random matrix $\left(A_{i j}\right)_{1 \leq i, j \leq d}$ distributed accordingly to this measure is a Gaussian Unitary Ensemble (GUE) random matrix.

We call $B=A-\frac{1}{d} \operatorname{Tr} A$ a traceless Gaussian Unitary Ensemble (GUE $E_{0}$ ) random matrix; it corresponds to the Gaussian probability measure on the set of $d \times d$ Hermitian matrices with trace zero and the density

$$
\frac{1}{Z_{d}^{\prime}} e^{-\frac{1}{2} \operatorname{Tr} H^{2}}
$$

with respect to the Lebesgue measure, where $Z_{d}^{\prime}$ is the normalizing constant.

The joint distribution of eigenvalues for GUE is well-known [Meh91, which allows us to find the corresponding distribution for $\mathrm{GUE}_{0}$; namely, if $x_{1} \geq \cdots \geq x_{d}$ are the eigenvalues of a $\mathrm{GUE}_{0}$ random matrix, then their joint distribution is supported on the hyperplane $x_{1}+\cdots+x_{d}=0$ with the density

$$
\frac{1}{C_{d}} e^{-\frac{x_{1}^{2}+\cdots+x_{d}^{2}}{2}} \prod_{i<j}\left(x_{i}-x_{j}\right)^{2}
$$

with respect to the Lebesgue measure, where $C_{d}$ is the normalization constant.

Theorem 3 (Main theorem). Let the integer $d \geq 1$ be fixed; for each $n \geq 1$ let $\lambda_{n}=\left(\lambda_{n, 1}, \ldots, \lambda_{n, d}\right)$ be, as in Section 1.1, a random Young diagram with $n$ boxes and with at most $d$ rows.

Then the joint distribution of the random variables $\left(\sqrt{\frac{2 d}{n}}\left(\lambda_{n, i}-\frac{n}{d}\right)\right)_{1 \leq i \leq d}$ converges, as $n \rightarrow \infty$, to the joint distribution of the eigenvalues of a GUE $E_{0}$ random matrix.

We postpone its proof to Section 2

Corollary 4. Let $d \geq 1$ be fixed, and for each $n \geq 1$ let $\pi_{n} \in S_{n}$ be a random permutation with the longest decreasing subsequence of length at most $d$. We denote by $\lambda_{n, 1}$ the length

of its longest increasing subsequence. Then the distribution of $\sqrt{\frac{2 d}{n}}\left(\lambda_{n, 1}-\frac{n}{d}\right)$ converges to the distribution of the largest eigenvalue of the GUE $E_{0}$ random matrix.

It should be pointed out that the distibution of eigenvalues of a $\mathrm{GUE}_{0}$ random matrix appears also in a related asymptotic problem [Joh01 of the distribution of the rows of a Young diagram associated (via RSK algorithm) to a random word consisting of $n$ letters in an alphabet of $d$ symbols in the limit of $n \rightarrow \infty$.

\subsection{Case $d=2$ revisited}

The set of $2 \times 2$ Hermitian matrices with trace zero can be viewed as a 3 -dimensional Euclidean space with a scalar product $\langle A, B\rangle=\operatorname{Tr} A B$. A $\mathrm{GUE}_{0}$ random matrix can be viewed under this correspondence as a Gaussian random vector in $\mathbb{R}^{3}$ the coordinates of which are independent with mean zero and variance 1 . Each $2 \times 2$ Hermitian traceless 
matrix $A$ has two eigenvalues $x_{1}=\lambda, x_{2}=-\lambda$, where $\lambda=\frac{1}{\sqrt{2}}\|A\|=\sqrt{\frac{\langle A, A\rangle}{2}}$. Therefore, for a $\mathrm{GUE}_{0}$ random matrix the corresponding random variable $2\left(x_{1}\right)^{2}$ is distributed like the square of the length of a standard Gaussian random vector in $\mathbb{R}^{3}$, which coincides

with the $\chi_{3}^{2}$ distribution; thus for $d=2$ Corollary 4 allows us to recover the result of Deutsch, Hildebrand and Wilf [DHW03].

\subsection{Idea of the proof}

In Section 2 we will prove Theorem 3, the main result of this article. Our proof will be based on an explicit calculation of the number of standard Young tableaux with a prescribed shape. The standard method to do this would be to use the hook-length formula, which would be not convenient for our purposes. Instead, we will use the determinantal formula of Frobenius and MacMahon. In order to make the connection to random matrices more explicit we shall recall its proof due to Zeilberger [Zei83 which is based on the observation that a Young tableaux with at most $d$ rows can be viewed as a certain trajectory of $d$ non-colliding particles on a line. Thus we will find explicitly the asymptotic joint distribution of the rows of a Young diagram; this distribution turns out to coincide with the distribution (11) of the eigenvalues of a $\mathrm{GUE}_{0}$ random matrix.

The reader may wonder if the connection between Young diagrams and random matrices given by Theorem 3 might be purely accidental. In the following paragraph we will argue why it is not the case and how deep connections between Young diagrams and random matrices may be seen in our proof of Theorem 3 .

In the above discussion we treated the distribution (10) of the eigenvalues of a $\mathrm{GUE}_{0}$ random matrix as granted; now let us think for a moment about its derivation. $\mathrm{GUE}_{0}$ is a Gaussian matrix; for this reason (up to a simple scaling factor) it can be viewed as a value at some fixed time of a matrix-valued Brownian bridge. It is known Dys62, Gra99. that the eigenvalues of a matrix-valued Brownion motion behave like Brownian motions conditioned not to collide. Since a matrix-valued Brownian bridge is a matrix-valued Brownian motion conditioned to be zero at time 1, it follows that its eigenvalues form Brownian motions conditioned not to collide and to be zero at time 1 ; in other words these eigenvalues form Brownian bridges conditioned not to collide. In this way the determinantal formula of Karlin and McGregor [KM59] can be applied. In the conditioning procedure we assume that the original positions of $d$ non-colliding particles are all different and we consider the limit as these initial positions converge to zero; in this way their final distribution is given by a continuous analogue of the formulas (10) and (8) which give the square of the number of Young tableaux of a given shape, with the transition probabilities replaced by the Gaussian kernels. One can easily check that such a derivation of the distribution of eigenvalues of a $\mathrm{GUE}_{0}$ random matrix follows (8) very closely.

To summarize: our proof of the main result will be based on the observation that both Young tableaux and the eigenvalues of matrix-valued Brownian motions can be interpreted as non-colliding particles and applying the determinantal formula of Karlin and McGregor [KM59]. 


\subsection{Final remarks}

We can see that both the case when $d$ is finite and the case considered in Section 1.3 corresponding to $d=\infty$ are asymptotically described by GUE random matrices. It would be very interesting to find a direct link between these two cases.

\section{Proof of the main result}

For a function $f: \mathbb{R} \rightarrow \mathbb{R}$ we define its difference $\Delta_{n} f: \mathbb{R} \rightarrow \mathbb{R}$ by

$$
\Delta_{n} f(y)=\frac{f\left(y+\sqrt{\frac{d}{n}}\right)-f(y)}{\sqrt{\frac{d}{n}}} .
$$

By iterating we define $\Delta_{n}^{\alpha} f$ for any integer $\alpha \geq 0$. We also define its shift $S_{n} f: \mathbb{R} \rightarrow \mathbb{R}$ by

$$
S_{n} f(y)=f\left(y+\sqrt{\frac{d}{n}}\right) .
$$

Notice that $S_{n}^{\alpha} f$ is well-defined for any integer $\alpha$.

Lemma 5. For each $n$ we define a function $f_{n}: \mathbb{R} \rightarrow \mathbb{R}$ which is constant on each interval of the form $\left[\frac{k-\frac{n}{d}}{\sqrt{\frac{n}{d}}}, \frac{k+1-\frac{n}{d}}{\sqrt{\frac{n}{d}}}\right)$ for each integer $k$ and such that

$$
f_{n}\left(\frac{k-\frac{n}{d}}{\sqrt{\frac{n}{d}}}\right)= \begin{cases}\sqrt{\frac{n}{d}} \frac{\left(\frac{n}{d}\right)^{k} e^{-\frac{n}{d}}}{k !} & \text { if } k \text { is a non-negative integer } \\ 0 & \text { if } k \text { is a negative integer. }\end{cases}
$$

Then for each integer $\alpha \geq 0$ and $y \in \mathbb{R}$

$$
\lim _{n \rightarrow \infty} \Delta_{n}^{\alpha} f_{n}(y)=\frac{d^{\alpha}}{d y^{\alpha}} \frac{1}{\sqrt{2 \pi}} e^{\frac{-y^{2}}{2}} .
$$

Furthermore, for each $\alpha \geq 0$ there exists a polynomial $P_{\alpha}$ such that

$$
\left|\Delta_{n}^{\alpha} f_{n}(y)\right|<P_{\alpha}(y) e^{-|y|}
$$

holds true for all $n$ and $y$.

Proof. Before presenting the proof we notice that $f_{n}$ is a density of a probability measure arising as follows: we normalize the Poisson distribution with the parameter $\nu=\frac{n}{d}$ in order to have mean 0 and variance 1 and we convolve it with a uniform distribution on the interval $\left[0, \sqrt{\frac{d}{n}}\right]$; therefore (3) states for $\alpha=0$ that the suitably rescaled probabilities of the Poisson distribution converge to the density of the normal distribution. The case $\alpha \geq 1$ shows that this convergence holds true also for differences (respectively, derivatives). 
The proof of (3) in the case $\alpha=0$ is a straightforward application of the Stirling approximation $\log z !=\left(z+\frac{1}{2}\right) \log z-z+\frac{\log 2 \pi}{2}+O\left(z^{-1}\right)$, namely for $y=\frac{k-\frac{n}{d}}{\sqrt{\frac{n}{d}}}$ such that $k$ is an integer we denote $c=\frac{n}{d}$. Then

$$
\begin{aligned}
& \log f_{n}(y)=\left(c+y \sqrt{c}+\frac{1}{2}\right) \log c-c-\log (c+y \sqrt{c}) != \\
& -\left(c+y \sqrt{c}+\frac{1}{2}\right) \log \left(1+\frac{y}{\sqrt{c}}\right)+y \sqrt{c}-\frac{\log 2 \pi}{2}+O\left(c^{-1}\right)=-\frac{y^{2}}{2}-\frac{\log 2 \pi}{2}+O\left(c^{-\frac{1}{2}}\right),
\end{aligned}
$$

where the above equalities hold true asymptotically for $y$ bounded and $c \rightarrow \infty$.

In order to treat the case $\alpha \geq 1$ we observe that the iterated derivative on the righthand side of (31) can be calculated by using the following three rules:

$$
\frac{d}{d y} e^{\frac{-y^{2}}{2}}=-y e^{\frac{-y^{2}}{2}} ; \quad \frac{d}{d y} y=1 ; \quad \frac{d}{d y}(\phi \psi)=\left(\frac{d}{d y} \phi\right) \psi+\phi \frac{d}{d y} \psi .
$$

Similarly, the iterated difference on the left-hand side of (3) can be calculated using the following three rules:

$$
\Delta_{n} f_{n}=-g_{n} S f_{n} ; \quad \Delta_{n} g_{n}=1 ; \quad \Delta_{n}(a b)=\left(\Delta_{n} a\right) b+\left(S_{n} a\right) \Delta_{n} b
$$

where $g_{n}: \mathbb{R} \rightarrow \mathbb{R}$ is a function which is constant on each interval of the form $\left[\frac{k-\frac{n}{d}}{\sqrt{\frac{n}{d}}}, \frac{k+1-\frac{n}{d}}{\sqrt{\frac{n}{d}}}\right)$ for each integer $k$ and such that

$$
g_{n}\left(\frac{k-\frac{n}{d}}{\sqrt{\frac{n}{d}}}\right)=\frac{k+1-\frac{n}{d}}{\sqrt{\frac{n}{d}}} .
$$

For each integer $\beta$ we have $\lim _{n \rightarrow \infty}\left(S_{n}^{\beta} f_{n}\right)(y)=\frac{1}{\sqrt{2 \pi}} e^{-\frac{y^{2}}{2}}$ and $\lim _{n \rightarrow \infty}\left(S_{n}^{\beta} g_{n}\right)(y)=y$ therefore each term contributing to the left-hand side of (3) converges to its counterpart on the right-hand side of (3), which finishes the proof of (3).

We consider $y=\frac{k-\frac{n}{d}}{\sqrt{\frac{n}{d}}}$; then

$$
\frac{\log f_{n}\left(\frac{k-\frac{n}{d}}{\sqrt{\frac{n}{d}}}\right)-\log f_{n}\left(\frac{k-1-\frac{n}{d}}{\sqrt{\frac{n}{d}}}\right)}{\sqrt{\frac{n}{d}}}=-\log \left(1+\frac{y}{\sqrt{\frac{n}{d}}}\right) \sqrt{\frac{n}{d}} .
$$

There is a constant $C_{1}<0$ with a property that if $y<C_{1}$ then the right-hand side of (515) is greater than 1 for any value of $n$. It follows that if $y_{i}=\frac{k_{i}-\frac{n}{d}}{\sqrt{\frac{n}{d}}}$ for $i \in\{1,2\}$ and $y_{1}<y_{2} \leq C_{1}$ then

$$
f_{n}\left(y_{1}\right) \leq f_{n}\left(y_{2}\right) e^{y_{1}-y_{2}} .
$$

Similarly we find a constant $C_{2}>0$ with a property that if $C_{2} \leq y_{1}<y_{2}$ then

$$
f_{n}\left(y_{2}\right) \leq f_{n}\left(y_{1}\right) e^{y_{1}-y_{2}} .
$$


For $\alpha=0$ inequality (4) holds true for $y$ in a small neighborhood of the interval $\left[C_{1}, C_{2}\right]$ for $P_{\alpha}$ being a sufficiently big constant which follows from (3) and compactness argument. Inequality (4) holds true outside of the interval $\left[C_{1}, C_{2}\right]$ by inequalities (6) and (17).

The case $\alpha \geq 1$ can be proved in an analogous way to the above proof of (3): we show that $\Delta_{n}^{\alpha} f_{n}$ is a sum of the terms of the form $\left(S_{n}^{\beta_{1}} g_{n}\right) \cdots\left(S_{n}^{\beta_{l}} g_{n}\right)\left(S_{n}^{\beta} f_{n}\right)$ and the absolute value of each such a term can be easily bounded by $P(y) e^{-|y|}$, where $P$ is a suitably chosen polynomial.

Proof of Theorem [3. The following discussion is based on the work of Zeilberger [Zei83]. Every Young tableau $T$ with at most $d$ rows and $n$ boxes can be interpreted as a trajectory of $d$ non-colliding particles $x_{1}(t), \ldots, x_{d}(t)$ on the real line as follows. We set

$$
x_{i}(t)=d+1-i+(\text { number of boxes of } T \text { in row } i \text { which are not bigger than } t) .
$$

In other words: the initial positions of the particles are given by $\left(x_{1}(0), \ldots, x_{d}(0)\right)=$ $(d, d-1, \ldots, 1)$. In each step one of the particles jumps to the right; the number of the particle which jumps in step $t$ is equal to the number of the row of the Young diagram $T$ which carries the box with a label $t$. The condition that $T$ is a standard Young tableau is equivalent to $x_{1}(t)>\cdots>x_{d}(t)$ for every value of $0 \leq t \leq n$.

Thus the results of Karlin and McGregor KM59 can be applied and the number of standard Young tableaux of the shape $\lambda_{1}, \ldots, \lambda_{d}$, where $|\lambda|=\lambda_{1}+\cdots+\lambda_{d}=n$, is equal to the determinant

$$
\begin{aligned}
& N_{\lambda_{1}, \ldots, \lambda_{n}}=n !\left|\begin{array}{cccc}
\frac{1}{\lambda_{1} !} & \frac{1}{\left(\lambda_{1}+1\right) !} & \cdots & \frac{1}{\left(\lambda_{1}+d-1\right) !} \\
\frac{1}{\left(\lambda_{2}-1\right) !} & \frac{1}{\lambda_{2} !} & \cdots & \frac{1}{\left(\lambda_{2}+d-2\right) !} \\
\vdots & \vdots & \ddots & \vdots \\
\frac{1}{\left(\lambda_{d}-d+1\right) !} & \frac{1}{\left(\lambda_{d}-d+2\right) !} & \cdots & \frac{1}{\lambda_{d} !}
\end{array}\right| \\
& =\frac{n ! e^{n}}{\left(\frac{n}{d}\right)^{n+\frac{d}{2}}}\left|\begin{array}{cccc}
f_{n}\left(y_{1}\right) & S_{n} f_{n}\left(y_{1}\right) & \cdots & S_{n}^{d-1} f_{n}\left(y_{1}\right) \\
S_{n}^{-1} f_{n}\left(y_{2}\right) & f_{n}\left(y_{2}\right) & \cdots & S_{n}^{d-2} f_{n}\left(y_{2}\right) \\
\vdots & \vdots & \ddots & \vdots \\
S_{n}^{-d+1} f_{n}\left(y_{d}\right) & S_{n}^{-d+2} f_{n}\left(y_{d}\right) & \cdots & f_{n}\left(y_{d}\right)
\end{array}\right| \\
& =\frac{n ! e^{n}}{\left(\frac{n}{d}\right)^{n+\frac{d(d+1)}{4}}}\left|\begin{array}{cccc}
f_{n}\left(y_{1}\right) & \Delta_{n} f_{n}\left(y_{1}\right) & \cdots & \Delta_{n}^{d-1} f_{n}\left(y_{1}\right) \\
S_{n}^{-1} f_{n}\left(y_{2}\right) & \Delta_{n} S_{n}^{-1} f_{n}\left(y_{2}\right) & \cdots & \Delta_{n}^{d-1} S_{n}^{-1} f_{n}\left(y_{2}\right) \\
\vdots & \vdots & \ddots & \vdots \\
S_{n}^{-d+1} f_{n}\left(y_{d}\right) & \Delta_{n} S_{n}^{-d+1} f_{n}\left(y_{d}\right) & \cdots & \Delta_{n}^{d-1} S_{n}^{-d+1} f_{n}\left(y_{d}\right)
\end{array}\right|,
\end{aligned}
$$

where

$$
y_{i}=\frac{\lambda_{i}-\frac{n}{d}}{\sqrt{\frac{n}{d}}}
$$

We are interested in a probability distribution on Young diagrams with $n$ boxes with the probability of $\left(\lambda_{1}, \ldots, \lambda_{d}\right)$ equal to

$$
\frac{1}{C_{n, d}}\left(N_{\lambda_{1}, \ldots, \lambda_{d}}\right)^{2},
$$


where $C_{n, d}$ is the suitably chosen normalizing constant. Clearly,

$$
\begin{gathered}
C_{n, d} \frac{\left(\frac{n}{d}\right)^{2 n+\frac{d^{2}+2 d-1}{2}}}{(n !)^{2} e^{2 n}}= \\
\sum_{\lambda_{1}, \ldots, \lambda_{d-1}}\left(\sqrt{\frac{n}{d}}\right)^{d-1}\left|\begin{array}{cccc}
f_{n}\left(y_{1}\right) & \Delta_{n} f_{n}\left(y_{1}\right) & \cdots & \Delta_{n}^{d-1} f_{n}\left(y_{1}\right) \\
S_{n}^{-1} f_{n}\left(y_{2}\right) & \Delta_{n} S_{n}^{-1} f_{n}\left(y_{2}\right) & \cdots & \Delta_{n}^{d-1} S_{n}^{-1} f_{n}\left(y_{2}\right) \\
\vdots & \vdots & \ddots & \vdots \\
S_{n}^{-d+1} f_{n}\left(y_{d}\right) & \Delta_{n} S_{n}^{-d+1} f_{n}\left(y_{d}\right) & \cdots & \Delta_{n}^{d-1} S_{n}^{-d+1} f_{n}\left(y_{d}\right)
\end{array}\right|^{2},
\end{gathered}
$$

where the sum runs over $\lambda_{1}, \ldots, \lambda_{d-1}$ such that for $\lambda_{d}=n-\left(\lambda_{1}+\cdots+\lambda_{d-1}\right)$ we have that $\lambda_{1}, \ldots, \lambda_{d}$ is a Young diagram with $n$ boxes. The right-hand side can be viewed as a Riemann sum; Lemma 5 shows that the dominated convergence theorem can be applied (with the dominating function of the form $P\left(y_{1}, \ldots, y_{d}\right) e^{-2\left(\left|y_{1}\right|+\cdots+\left|y_{d}\right|\right)}$, where $P$ is some polynomial) and

$$
\lim _{n \rightarrow \infty} C_{n, d} \frac{\left(\frac{n}{d}\right)^{2 n+\frac{d^{2}+2 d-1}{2}}}{(n !)^{2} e^{2 n}}=\int_{y_{1}, \ldots, y_{d-1}}\left|\begin{array}{cccc}
e^{-\frac{y_{1}^{2}}{2}} & \frac{d}{d y_{1}} e^{-\frac{y_{1}^{2}}{2}} & \cdots & \frac{d^{d-1}}{d y_{1}^{d-1}} e^{-\frac{y_{1}^{2}}{2}} \\
e^{-\frac{y_{2}^{2}}{2}} & \frac{d}{d y_{2}} e^{-\frac{y_{2}^{2}}{2}} & \cdots & \frac{d^{d-1}}{d y_{2}^{d-1}} e^{-\frac{y_{2}^{2}}{2}} \\
\vdots & \vdots & \ddots & \vdots \\
e^{-\frac{y_{d}^{2}}{2}} & \frac{d}{d y_{d}} e^{-\frac{y_{d}^{2}}{2}} & \cdots & \frac{d^{d-1}}{d y_{d}^{d-1}} e^{-\frac{y_{d}^{2}}{2}}
\end{array}\right|^{2} d y_{1} \cdots d y_{d-1},
$$

where the integral runs over $\left(y_{1}, \ldots, y_{d-1}\right)$ such that for $y_{d}=-\left(y_{1}+\cdots+y_{d-1}\right)$ we have $y_{1} \geq \cdots \geq y_{d}$.

Since the limit density defines a probability measure, in the limit $n \rightarrow \infty$ the random variables $\left(y_{1}, \ldots, y_{d-1}\right)$ (please notice that due to the constraint $y_{1}+\cdots+y_{d}=0$ the value of $y_{d}$ is uniquely determined by $\left.y_{1}, \ldots, y_{d-1}\right)$ converge in distribution to the probability measure on the set $y_{1} \geq y_{2} \geq \cdots \geq y_{d-1} \geq-\left(y_{1}+\cdots+y_{d-1}\right)$ with a density

$$
\frac{1}{C_{d}^{\prime}}\left|\begin{array}{cccc}
e^{-\frac{y_{1}^{2}}{2}} & \frac{d}{d y_{1}} e^{-\frac{y_{1}^{2}}{2}} & \cdots & \frac{d^{d-1}}{d y_{1}^{d-1}} e^{-\frac{y_{1}^{2}}{2}} \\
e^{-\frac{y_{2}^{2}}{2}} & \frac{d}{d y_{2}} e^{-\frac{y_{2}^{2}}{2}} & \cdots & \frac{d^{d-1}}{d y_{2}^{d-1}} e^{-\frac{y_{2}^{2}}{2}} \\
\vdots & \vdots & \ddots & \vdots \\
e^{-\frac{y_{d}^{2}}{2}} & \frac{d}{d y_{d}} e^{-\frac{y_{d}^{2}}{2}} & \cdots & \frac{d^{d-1}}{d y_{d}^{d-1}} e^{-\frac{y_{d}^{2}}{2}}
\end{array}\right|^{2}=\frac{1}{C_{d}^{\prime}}\left|\begin{array}{cccc}
p_{0}\left(y_{1}\right) e^{-\frac{y_{1}^{2}}{2}} & p_{1}\left(y_{1}\right) e^{-\frac{y_{1}^{2}}{2}} & \cdots & p_{d-1}\left(y_{1}\right) e^{-\frac{y_{1}^{2}}{2}} \\
p_{0}\left(y_{2}\right) e^{-\frac{y_{2}^{2}}{2}} & p_{1}\left(y_{2}\right) e^{-\frac{y_{2}^{2}}{2}} & \cdots & p_{d-1}\left(y_{2}\right) e^{-\frac{y_{2}^{2}}{2}} \\
\vdots & \vdots & \ddots & \vdots \\
p_{0}\left(y_{d}\right) e^{-\frac{y_{d}^{2}}{2}} & p_{1}\left(y_{d}\right) e^{-\frac{y_{d}^{2}}{2}} & \cdots & p_{d-1}\left(y_{d}\right) e^{-\frac{y_{d}^{2}}{2}}
\end{array}\right|^{\cdots}
$$

for a suitably chosen normalizing constant $C_{d}^{\prime}$, where $\frac{d^{k}}{d z^{k}} e^{-\frac{z^{2}}{2}}=p_{k}(z) e^{-\frac{z^{2}}{2}}$ for some polynomial $p_{k}$ (related to Hermite polynomials). Since $p_{k}(z)=(-z)^{k}+$ (summands of lower degree) the above expression takes a simpler form: 


$$
\frac{1}{C_{d}^{\prime}}\left|\begin{array}{cccc}
e^{-\frac{y_{1}^{2}}{2}} & \left(-y_{1}\right) e^{-\frac{y_{1}^{2}}{2}} & \cdots & \left(-y_{1}\right)^{d-1} e^{-\frac{y_{1}^{2}}{2}} \\
e^{-\frac{y_{2}^{2}}{2}} & \left(-y_{2}\right) e^{-\frac{y_{2}^{2}}{2}} & \cdots & \left(-y_{2}\right)^{d-1} e^{-\frac{y_{2}^{2}}{2}} \\
\vdots & \vdots & \ddots & \vdots \\
e^{-\frac{y_{d}^{2}}{2}} & \left(-y_{d}\right) e^{-\frac{y_{d}^{2}}{2}} & \cdots & \left(-y_{d}\right)^{d-1} e^{-\frac{y_{d}^{2}}{2}}
\end{array}\right|^{2}=\frac{1}{C_{d}^{\prime}} e^{-\left(y_{1}^{2}+\cdots+y_{d}^{2}\right)} \prod_{1 \leq i<j \leq d}\left(y_{i}-y_{j}\right)^{2}
$$

When we set $x_{i}=\sqrt{2} y_{i}=\sqrt{\frac{2 d}{n}}\left(\lambda_{n, i}-\frac{n}{d}\right)$ it becomes clear that the limit distribution of $\left(x_{1}, \ldots, x_{d}\right)$ coincides with the distribution (11) of the eigenvalues of a $G U E_{0}$ random matrix, which finishes the proof.

Acknowledgements: The research was performed during a visit to Queens University. I thank Jonathan Novak for many discussions and for pointing out the reference [DHW03]. I thank Roland Speicher for invitation and hospitality during this stay.

Research supported by the MNiSW research grant 1 P03A 013 30, by the EU Research Training Network 'QP-Applications', contract HPRN-CT-2002-00279 and by the EC Marie Curie Host Fellowship for the Transfer of Knowledge 'Harmonic Analysis, Nonlinear Analysis and Probability', contract MTKD-CT-2004-013389.

\section{References}

[BDJ99] Jinho Baik, Percy Deift, and Kurt Johansson. On the distribution of the length of the longest increasing subsequence of random permutations. J. Amer. Math. Soc., 12(4):1119-1178, 1999.

[DHW03] Emeric Deutsch, A. J. Hildebrand, and Herbert S. Wilf. Longest increasing subsequences in pattern-restricted permutations. Electron. J. Combin., 9(2):Research paper 12, 8 pp. (electronic), 2002/03.

[Dys62] Freeman J. Dyson. A Brownian-motion model for the eigenvalues of a random matrix. J. Mathematical Phys., 3:1191-1198, 1962.

[Gra99] David J. Grabiner. Brownian motion in a Weyl chamber, non-colliding particles, and random matrices. Ann. Inst. H. Poincaré Probab. Statist., 35(2):177204, 1999.

[Joh01] Kurt Johansson. Discrete orthogonal polynomial ensembles and the Plancherel measure. Ann. of Math. (2), 153(1):259-296, 2001.

[KM59] Samuel Karlin and James McGregor. Coincidence probabilities. Pacific J. Math., 9:1141-1164, 1959.

[Meh91] Madan Lal Mehta. Random matrices (2 ${ }^{\text {nd }}$ ed.). Academic Press, Boston, 1991.

[Oko00] Andrei Okounkov. Random matrices and random permutations. Internat. Math. Res. Notices, (20):1043-1095, 2000.

[Zei83] Doron Zeilberger. André's reflection proof generalized to the many-candidate ballot problem. Discrete Math., 44(3):325-326, 1983. 